\title{
SEMINARIO SOBRE LA PARTICIPACION DE LOS PADRES Y DE LA COMUNIDAD EN LA EDUCACION INFANTIL TEMPRANA
}

\author{
Tercer Seminario del Hemisferio Occidental
}

En la ciudad de Cali se llevó a cabo entre el 20 y el 31 de marzo el Tercer Seminario sobre Educación Infantil Temprana en el Hemisferio Occidental, promovido por la Fundación Bernard Van Leer, la cual patrocina investigaciones y programas tendientes al estudio de la atención infantil temprana especialmente en la población socialmente deprivada.

Dicho Seminario estuvo coordinanado por: CIVAL (Comité de Integración de Proyectos de la Fundación Bernard Van Leer en Colombia) del cual hace parte el Centro de Investigaciones de la Universidad Pedagógica Nacional -CIUP_.

Participaron los países que están actualmente realizando investigaciones en este campo como: Brasil, Colombia, Chile, Escocia, España, Estados Unidos, Holanda, Jamaica, Kenja, Malasia, Perú y Reino Unido.

Los temas tratados durante el Seminario fueron:

La Familia, primera fuente de cuidado y educación, la escuela, canal inicial que vincula al niño con el sonido exterior, la comunidad, extensión de la familia para el desarrollo educacional y social; y la participación integrada en los procesos de cambio, mirando hacia el porvenir.

Las conclusiones del Seminario se han considerado muy importantes, por tal razón se presenta a la comunidad educativa una síntesis de dichas conclusiones las cuales serán publicadas en su totalidad posteriormente.

\section{CONCLUSIONES}

\section{A. La Familia: Una fuente primaria de cuidado y educación}

—Unánimemente se aceptó que la familia constituye "el núcleo y la célula primaria de organización social", pero al mismo tiempo se hizo énfasis que en la práctica existen muchas formas de la expresión familia, especialmente en el contexto multicultural de la región.

—La expresión "familia" se utilizó únicamente como un marco de referencia básico. La influencia del padre o la madre puede ser muy diferente, de acuerdo con los distintos contextos sociales. A medida que se desciende en la escala social, estas influencias pueden llegar a ser gradualmente negativas en términos del desarrollo del niño.

- El trabajo físico de la madre, necesario en la mayoría de las comunidades, demanda una gran cantidad de calorías que ella no está en capacidad de satisfacer. De ahí que desde el momento de la concepción el niño empiece a sufrir las consecuencias de su condición marginal. 
- La disminución de la lactancia materna, con las consecuencias negativas de privar al infante del calor humano necesario, puede resultar también en el empleo de alimentos pobres en proteínas y otros nutrientes.

-El número, constantemente en aumento, de mujeres que trabajan por fuera del hogar, plantea problemas especiales tales como obligar a otros miembros de la familia, particularmente al hombre (cuando está presente) o a la comunidad, a asumir las funciones del cuidado del niño. Como consecuencia, la tendencia universal en los países en desarrollo, de excluir al hombre de las actividades del cuidado del niño, está cambiando, y trae consigo un nuevo juego de percepciones acerca de los papeles masculino y femenino.

-El nivel de educación de los padres parece ser un factor significativo en la determinación de la estructura y la calidad del ambiente en el cual el niño vive y aprende. Un enfoque necesario, pero no suficiente, para responder a este problema, es educar a los padres, particularmente a la madre, en principios modernos de higiene, salud y nutrición.

- Los futuros padres pueden ser preparados muy bien para sus papeles, a través de programas específicos en el currículo formal y más todavía, en forma especial, permitiendo a los niños y adolescentes interactuar con otros niños y adultos en actividades de cuidado responsable de los niños, en ambientes concretos que permitan adquirir conocimientos de relaciones, conflictos y resolución de problemas.

-Respecto a la posibilidad de desarrollar programas de educación para padres pertenecientes a zonas marginadas, se concluyó que podría llegarse después de un cuidadoso análisis, al diseño de estrategias de supervivencia y/o desarrollo, acordes con las necesidades de cada familia y de cada niño.

-Es necesario establecer programas de planificación familiar, pero, al hacerlo, debe prestarse gran atención a la sensibilidad cultural de los usuarios, como también tener en cuenta las políticas nacionales de planificación familiar aunque los programas innovadores no deben obligatoriamente adecuarse a ellas.

-Una de las consecuencias del fenómeno de la migración ha sido la ruptura de los patrones tradicionales de la familia extendida, y de otros soportes que han pretendido ofrecer alguna solidaridad social y apoyo en las áreas rurales tradicionales. El derrumbe de las instituciones sociales ha estimulado el surgimiento de condiciones sociales "patológicas". En el caso particular de Colombia, el problema del "gamin" o niño abandonado, es particularmente notorio. El fenómeno no es, sin embargo, exclusivo de este país y tiene múltiples manifestaciones equivalentes en todas las naciones en desarrollo, en el mundo entero, donde las formas tradicionales de "estructura familiar", están bajo presión. Las consecuencias sociales negativas —criminalidad infantil, prostitución juvenil y otras-, son un producto inevitable de esta situación acumulativa y parecen tener relación directa con la desintegración de las formas establecidas de familia y las estructuras coherentes de apoyo tradicional.

-Respecto al problema anterior existe, en general, una marcada tendencia a organizar programas especiales para trabajar con la expresión sintomática del problema, cuando los niños ya están en edad pre-escolar y, aún en la escuela primaria. 
Vale la pena destacar la evolución del programa de la Fundación de Investigaciones de Ecología Humana de la ciudad de Cali, Colombia. El proyecto de nutrición con el cual se inició, produjo mejoras significativas entre niños marginados de edad preescolar, especialmente en el área del desarrollo físico; sin embargo, más adelante fue necesario enriquecerlo y para esto se acudió a un enfoque multidimensional que ofrecía nutrición, cuidados en salud y educación pre-escolar. En etapas posteriores se hizo necesario incluir educación para padres de familia y adultos encargados de los niños, con el propósito de demostrar cómo el ambiente inmediato, la familia que rodea al niño, puede, a pesar de circunstancias económicas negativas, modificarse para proveer un contexto mejor para el crecimiento y desarrollo del niño.

El cambio fue de un enfoque en patologías y síntomas, a un enfoque preventivo, constructivo y educativo.

-En los países del sector andino, en América Latina, se observan problemas ambientales muy específicos, como consecuencia de condiciones ecológicas que varían de acuerdo con la altura, y los factores étnicos, culturales y fisiológicos. Los patrones familiares también son marcadamente diferentes.

-Esto sugiere la realización de programas experimentales con familias de los tres principales estratos socio-culturales y sistemas ecológicos de la región: la población predominante negra de la Costa, la población mestiza de las alturas medias y la población indígena de las grandes alturas. Sólo de esta manera se puede esperar que surjan prácticas efectivas para la educación y apoyo de las diferentes familias de la región.

-Aún cuando se hizo gran énfasis en la necesidad de respetar las diferencias culturales, se identificaron algunos principios generales para la educación de los padres y la familia: En la cultura de pobreza existe una mayor preocupación por la educación de las madres, dada la función que ella tiene que cumplir respecto al cuidado y educación de los hijos. A pesar de que el padre es un miembro que permanece muy poco tiempo en el hogar debe hacerse los esfuerzos que sean necesarios para lograr su participación en el cuidado del niño. En el caso de grupos u organizaciones que funcionen como padres substitutos deben tener muy clara la necesidad del niño de recibir la influencia masculina y femenina tan importante en los primeros años.

—Se debe discutir si el "centro de gravedad" social debe ser la familia o más bien lo que el niño percibe y experimenta, y cómo interpreta su ambiente e interacción con él.

-Respecto al lenguaje se percibió que las familias marginadas tienen un lenguaje pobre y lo usan en forma muy simple. En comparación con otras formas de comunicación, el, lenguaje es poco elaborado y secundario. El lenguaje del niño no se enriquece en su contacto con los adultos. Con muy poca frecuencia se le hacen preguntas, y el diálogo casi no existe. Considerando experiencias anteriores se acordó que este aspecto sería mejor tratado utilizando estrategias a nivel del hogar.

- Dentro del contexto de deprivación especialmente en el área del lenguaje, es importante considerar cuidadosamente la diferencia entre deficiente y diferente. El lenguaje que puede ser considerado, en términos de uso común, deficiente, puede ser completamente efectivo en su contexto. La deficiencia en estas circunstancias se refiere sencillamente a diferencia en el estilo de lenguaje. 
-En muchas sociedades la familia extendida es una fuerza conservadora fuerte, que actúa como freno para cambios progresistas. En muchos países de la región, la evidencia de esto se ve en los millones de personas que cambian la "seguridad" y el soporte de su familia extendida y las áreas rurales por las inseguridades de la ciudad. Se puede pensar que esta iniciativa (aun cuando sus motivaciones son múltiples), está rompiendo los viejos esquemas de soporte, y de hecho provee una oportunidad para cambios favorables. Es una condición que se desarrolla en programas para la elaboración de nuevos sistemas de soporte, nuevas formas de "familia" más aptas para las nuevas circunstancias. Para hacer esto se ve otra vez la necesidad de introducir modelos educativos con bases amplias que abarquen una estructura múltiple de factores interactuantes y cooperativos dirigidos al desarrollo, ajustable a condiciones de cambio, más que un intento "compensatorio" y remedial para reestablecer algún tipo de status idealizado "quo ante".

-Para desarrollar programas realmente efectivos y duraderos, es necesario que provengan de una unión genuina entre todas las fuentes posibles de autoridad y poder que influyen en el niño, tales como la familia, el maestro, los líderes de la comunidad, la educación oficial y otros.

\section{B. La Escuela: el canal inicial entre el niño y el mundo exterior}

-Dentro del amplio tema "los padres y la participación comunitaria en la educación infantil" uno de los aspectos que se plantean es si la escuela puede o no, actuar como agente de cambio y hasta qué punto, particularmente en comunidades marginadas.

-Respecto a la "educación compensatoria" se concluyó que este término debe ser usado muy cuidadosamente para evitar actitudes paternalistas por parte de los maestros tradicionales que carecen de capacitación y motivación hacia una dimensión más amplia de la educación. Su acción a nivel pre-escolar puede ser adecuada pero no debe limitarse al contexto institucional sino ir más allá, incluyendo la familia.

- La escuela puede considerarse como uno entre otros instrumentos para la preparación de niños, maestros y comunidad, especialmente en la modificación de actitudes, no obstante las limitaciones de esta acción.

-El punto anterior plantea el problema de los objetivos: se buscan, a largo plazo, resultados de índole socio-afectiva tanto como cognoscitiva, o resultados, a corto plazo, de adaptación del niño al sistema existente?

La respuesta práctica, sobre todo para los países latinoamericanos y del Caribe, se encuentra, seguramente, en un punto medio; pero saber exactamente dónde, requiere un cuidadoso estudio, ya que la elección afectará profundamente aspectos tales como el sistema de educación y los currículos, con todas sus implicaciones.

-Dentro del marco social dominante en la región, los educadores tienen el compromiso de contribuir a una concientización social aun cuando en muchas situaciones no se vislumbren cambios en un futuro inmediato, y aún cuando se argumente que desde un punto de vista práctico es más importante promover la toma de conciencia entre aquellos que están aplicando los programas innovativos que entre quienes los diseñan.

-El entrenamiento de los maestros en los países de la región tiene grandes alcances: Los programas deben contemplar la problemática de las comunidades marginadas y 
buscar el compromiso de los maestros para dar una ayuda práctica a los niños y a los padres en la solución de sus problemas.

Actualmente la preparación de maestros depende primordialmente de modelos culturales y de contenidos importados de Europa y Norte América. Muchos de esos modelos no incluyen el aspecto de la formación de actitudes hacia la comunidad de la cual se trata aquí, ni la necesidad de que la educación considere los aspectos básicos de supervivencia en circunstancias de deprivación.

-El momento actual no parece adecuado para hacer mucho énfasis en la "escuela y comunidad ideal".

Teniendo en cuenta la carencia de actitudes deseables, la unión entre escuela y comunidad quizá no sea realizable en un futuro cercano, pero debe fomentarse esta noción a pesar de ser una tarea lenta y difícil; especialmente en comunidades marginadas esto se ha trabajado en forma muy superficial, dando la impresión de participación pero sin existir una verdadera responsabilidad y el derecho a tomar decisiones.

- La escuela, en cuanto sea posible, debe estar en armonía visual y arquitectónica con el ambiente específico y no simbolizar un mundo aparte, donde el niño se sienta como un visitante incómodo, ni diseñarse, como en ciertos casos, con el fin de deslumbrar al niño y su familia, ante la maquinaria gubernamental.

- La respuesta a las necesidades reales del niño marginado implica la necesidad de modificar deliberadamente al patrón de la escuela como instrumento que satisface las demandas de la sociedad.

- Se aceptó que a nivel mundial existe consenso sobre la importancia de la educación dentro de los planes de desarrollo social, la importancia de la cooperación con los padres y la participación de la comunidad, como también sobre lo arraigados y defensivos que son los sistemas educativos. Lo anterior ha llevado a muchas personas interesadas en la innovación, a rechazar las instituciones de educación formal y a poner todas sus esperanzas en las iniciativas de carácter informal.

- Una de las razones para mirar fuera del marco convencional y buscar nuevas agencias educativas que puedan satisfacer en forma más eficiente las necesidades de los niños marginados radica en las limitaciones y resistencia de muchos de los sistemas actuales. Aún en aquellos casos donde el sistema tradicional es razonablemente bueno, no será capaz, por lo menos a corto plazo, de atender los cambios radicales necesarios. Es significativo el hecho que la región ha producido una amplia gama de formas institucionales interesantes, fuera del marco convencional escolar, las cuales se relacionan con la necesidad general de que existan medios efectivos de bajo costo para el niño marginado. Las "Casas Vecinales" de Colombia, las "Escuelas Básicas" de Jamaica, las "Escuelas Alternas para la Educación Vocacional" de Trinidad, son ejemplos de un intento planificado de complementación a la escuela y de apartarse del formalismo de las estructuras convencionales de la educación.

-Respecto a la generación de enlaces entre los padres y la comunidad y estos dos grupos y la escuela, se destacó la experiencia de Jamaica, la cual, aún en los casos de mayor deprivación, ha progresado en forma secuencial, desde asistencia a asociación y finalmente a coparticipación. 
En las etapas finales, los maestros, los padres y otros trabajadores se han educado mutuamente hasta el punto de estar comprometidos en el planteamiento conjunto de programas para el desarrollo integral del niño. En este caso se llegó a una interacción satisfactoria entre la escuela y la comunidad; sin embargo se puso de relieve que así como no puede esperarse que los maestros se desempeñen efectivamente por fuera de las circunstancias que les son familiares sin haber recibido una preparación adecuada, igualmente la comunidad debe ser reforzada progresivamente en el cumplimiento de las etapas necesarias para su participación.

-Probablemente la innovación más significativa que ha producido la región es la utilización de para-profesionales en educación.

Estos trabajadores introducen en el contexto de la escuela una dimensión totalmente nueva, mayor familiaridad con la comunidad y sus realidades, sensibilidad a las culturas locales, conciencia de las experiencias de la vida real. Ellos, a través de su vinculación a la economía y la cultura, capacitan la escuela para responder más efectivamente a las demandas de la comunidad: Los así llamados para-profesionales son, en muchas formas, exponentes profesionales de la cultura particular y estilos de vida y contribuyen en sus diferentes formas individuales, a la satisfacción de las necesidades del niño en su propia comunidad. Ellos ponen a disposición de la educación toda una nueva gama de talentos, discernimientos y percepciones.

—Los proyectos de la Fundación en Colombia, Jamaica, Trinidad y Venezuela, por distintos caminos y contextos, han demostrado la fuerza y el valor especial de este innovativo recurso. A partir de esta experiencia parecería que el sistema preescolar más exitoso y práctico, es uno en el cual unos pocos profesionales, altamente entrenados y experimentados, se hagan cargo del entrenamiento y del seguimiento de numerosos paraprofesionales que sean los verdaderos trabajadores de la escuela. Tal sistema tiene ventajas muy concretas sobre el sistema profesionalizado: Económicamente es consecuente con los recursos de un país pobre. Los trabajadores de la escuela son escogidos, si no entre la misma comunidad, por lo menos entre personas de contextos sociales similares a los de los niños. Así, estos interactuarán con adultos cuya imagen no es muy diferente de la de sus padres.

\section{La Comunidad: Una familia extendida para el desarrollo social y económico}

—La noción de "Comunidad" vista como una "Familia Extensa" que apoya la educación infantil, se relaciona. con el hecho de cómo un enfoque educativo particular puede derivar aspectos importantes del ambiente físico y social donde se desarrolla. Recíprocamente, también se relaciona con la forma como el aprendizaje de las adultos se puede incrementar fuera de las estructuras formales, al tomar conciencia del conocimiento que existe en la actualidad en el área de educación y desarrollo infantil. Muchos grupos de comunidad están ubicados en ambientes que, a pesar de ser desalentadores social y económicamente, proveen una amplia gama de oportunidades de aprendizaje estimulante y de situaciones para resolver problemas que reflejan asuntos vitales que repercuten de inmediato en la vida de los individuos involucrados. Es esta confrontación con la realidad la que convierte la comunidad en un recurso educativo, especialmente interesante en los contextos de marginalidad y deprivación. Pero, existe un asunto metodológicamente básico relacionado con este aspecto. En educación comunitaria el educando es confrontado con problemas sobre los cuales debe reflexionar y cuya resolución debe buscar por sí mismo. El concepto y estilo implícitos son los mismos del "aprendizaje por descubrimiento", o "no directivo". Al educando se le orienta a adquirir conocimientos y 
ganar experiencias por sí mismo y llegar a sus propias soluciones antes que aceptar las soluciones de otros.

— La prueba de que una comunidad funciona está en su habilidad para facilitar el desarrollo de aquellas personas que la integran.

- Se da por hecho que quienes están involucrados en educación comunitaria tiene que "empezar donde está la gente"; pero también deben contemplar las mejores alternativas y realidades que van más allá de aquellas que ya existen y en las cuales las habilidades y capacidades de las comunidades involucradas se mejoran de tal manera que pueden edificar y controlar su futuro de una manera creativa.

- La fórmula comunidad-escuela, como un centro de educación formal y no formal, ha tenido éxitos en algunas áreas y evita la tendencia a desarrollar sistemas fragmentarios y opuestos.

— Las actividades prácticas comunitarias que responden a necesidades inmediatas y su adecuación a la vida diaria de los individuos, promueven toma de conciencia personal y preocupación por los demás, cualidades esenciales para el crecimiento personal y el desarrollo.

-El proceso de organizar una comunidad alrededor de sus necesidades concretas para la toma de decisiones y la acción de colaboración, provee un marco de referencia para las comunidades en transición y las comunidades en desarrollo.

- Las circunstancias probablemente determinarán si una comunidad particular puede beneficiarse de un programa que haya surgido y se haya implementado en otra comunidad, o, si el proyecto debe ser original y derivado de los propósitos y estilo de esa comunidad en particular.

-El liderazgo en sí mismo altera la dinámica de la comunidad; por lo tanto, es importante que el líder perciba las necesidades de la comunidad, a través del contacto directo con la gente, más que intentando imponer sus propios puntos de vista acerca de los cambios necesarios. También debe recordarse que la mayoría de las comunidades marginales comparten promesas incumplidas; por lo tanto los agentes externos deben abstenerse de adquirir compromisos que excedan lo que honestamente pueda ser cumplido.

-Dos aspectos son de especial importancia en el intento de asegurar la continuación de un programa: Uno es la identificación y entrenamiento de personas de la comunidad que puedan asumir posiciones de liderazgo dentro de la comunidad y dentro del programa, antes que termine la acción del agente externo. El segundo es la compatibilidad, cuando sea razonable, con los intereses y las políticas nacionales.

A pesar de que muy probablemente programas innovadores no son transferibles a ambientes culturales y marcos temporales para los que inicialmente se diseñara el programa, la divulgación de información acerca de diferentes elementos del programa y del proceso de desarrollo, es útil más allá de un ambiente determinado.

- Con frecuencia uno de los aspectos más difíciles en las fases de organización e intervención en la comunidad es cómo seleccionarla. Una comodidad que sea claramente típica en un país, puede aparentemente ofrecer mayor posibilidad de generalización posterior. Pero, se señaló que una comunidad atípica, puede tener otra ventaja que es la 
de iluminar factores de desarrollo importantes, menos notorios en un ambiente típico. También se formuló la pregunta, desde el punto de vista del grado de desarrollo, si es mejor seleccionar una comunidad que ya posea los rudimentos de las estructuras comunitarias y construir de allí en adelante, o si es preferible escoger un grupo de gente que aún no se ha organizado o identificado sus intereses comunes y sus problemas. Ninguna conclusión general puede darse a esta pregunta. Tales decisiones se originan en sistemas de prioridades locales y personales, en recursos e intereses.

- Igualmente, al tratar con comunidades marginadas que presentan frecuentemente resistencia al agente externo, es importante tener en cuenta que hay momentos de madurez en los cuales la idea de desarrollo puede ser introducida más eficazmente. La técnica de promover y manejar con éxito un proyecto de comunidad, depende, en gran medida, de la capacidad para identificar estos momentos vitales. En síntesis, es importante encontrar el equilibrio entre las necesidades sentidas, la acción tradicional del trabajador comunitario y aquellas necesidades que han sido percibidas por el agente externo, con una orientación hacia el desarrollo.

-En los contextos absolutamente locales y regionales, una preocupación importante es la implicación política y social de los programas de acción comunitaria. Actualmente el desarrollo se entiende en un sentido más amplio y dinámico que en los años sesenta. Es importante, sin embargo, relacionar proyectos experimentales de este tipo con planes y programas nacionales apropiados. La acción social articulada con políticas nacionales de planeamiento, gana poder y significación y sostiene la posibilidad de la influencia reflexiva del programa en la política y viceversa. Este hecho es de particular importancia para América Latina.

-Respecto al entrenamiento del personal para proyectos comunitarios, la experiencia en Colombia subraya la necesidad de grupos interdisciplinarios con una gran dosis de colaboración de los participantes y con un estilo de trabajo de seminario para relacionar la teoría con la experiencia.

-A pesar que no se ofrecieron líneas claras e inequívocas para la operacionalización de proyectos comunitarios, surgieron algunos principios generales. Por ejemplo, se señaló que la intervención externa debe ocurrir solamente como respuesta a una solicitud específica de una comunidad.

-En general, el éxito de los proyectos comunitarios dentro de la región no fue muy relevante salvo pocas excepciones. La aceptación de ideas y prácticas innovadoras por parte de los sistemas establecidos apareció como un problema constante y se advirtió además, la tendencia a realizar ellos mismos, desde adentro, las innovaciones.

-Tanto la resistencia de grupos locales a la intervención de la burocracia como la oposición de ésta al cambio, son diferentes aspectos del mismo síndrome. Solamente si ambos sectores se involucran activamente en el proceso de planear y controlar el cambio, pueden esperarse resultados satisfactorios.

- Se discutió ampliamente el punto si la escuela puede o no hacer una contribución en el contexto de educación comunitaria y si la respuesta es afirmativa, cuales serian, entonces, las condiciones para convertir la teoría en práctica. Se hace necesario un cambio de actitudes en los maestros y en los padres para lograr la integración escuelacomunidad que generalmente conduce a la "comunidad" que funciona como una familia extensa para el desarrollo social. 
- La escuela constituye en la actualidad la única institución potencialmente capaz de tener contacto con todas las familias a través de los niños durante los años críticos de su desarrollo. De otra parte, las familias de las áreas marginadas necesitan ayuda para cumplir sus diferentes funciones básicas y tener acceso a una serie de facilidades y recursos que existen; es decir, necesitan una ayuda y orientación que puede ser asumida por la escuela. De esta manera, ella puede actuar como una guía valiosa y mediadora enseñando la forma de utilizar los servicios existentes y colaborando a que aquellos que los prestan entiendan cómo deben responder de una manera adecuada a las necesidades de los niños, las familias y la comunidad.

- Considerando la cantidad y la magnitud de los problemas de las comunidades marginadas, se requiere examinar su potencial para construir respuestas propias actuando sobre los principios de auto-ayuda, los cuales han surgido en diferentes países en desarrollo. Clave para esto es la detección del líder local y los problemas de financiación, tanto durante la etapa de intervención externa como una vez que ésta finalice.

- Finalmente, una función de la educación en comunidad podría ser motivar y capacitar comunidades mediante técnicas de auto-ayuda para desarrollar sus propias estrategias de supervivencia.

\section{Mirando hacia el porvenir: Participación integrada de los procesos de cambio}

-Es necesario mantener una versión optimista del futuro, pese a los muchos indicadores que podrían sugerir lo contrario.

- Producir el cambio en instituciones o comunidades enraizadas en las formas tradicionales es un asunto lento y delicado. No se puede esperar que los resultados aparezcan rápidamente y es muy importante que se establezcan plazos realistas a pesar de la impaciencia de las autoridades nacionales.

- La prioridad concedida en la mayoría de los países en desarrollo a programas de educación infantil temprana, obedece a que se consideran esenciales para el bienestar de la sociedad. Ellos constituyen un vehículo importante en la construcción progresiva del capital humano.

—En la búsqueda de "soluciones" a las complejas necesidades de las comunidades en desarrollo, es claro que un enfoque educativo solo es insuficiente. Las soluciones deben ser amplias y relativas a grupos de problemas que afectan aspectos de la vida de una comunidad. Esas soluciones deben tener en cuenta aspectos tales como la salud y su cuidado, saneamiento, nutrición, principios de organización de la comunidad. En casos así, el papel y el peso de la educación, en cualquiera de sus formas, depende de la apreciación inmediata de los requerimientos de cada caso específico.

— La inclusión dentro de los programas experimentales de desarrollo de diferentes componentes de servicio, actúa sinergísticamente en el impacto total. El impacto de un todo integrado que se adecua razonablemente a la situación, es diferente y mayor que la suma de las partes individuales.

— La realidad de hacer cambios reales en el futuro, en esas comunidades, descansa sobre los hombros de "pequeñas gentes haciendo pequeñas cosas". La noción del cambio 
acumulativo se aprecia como una iniciativa con muchas más posibilidades de éxito, que la de las ideas espectaculares.

-El papel de las intervenciones externas es más exitoso si se orienta a ayudar a la gente a determinar sus propias rutas, generar sus propias técnicas y procesos y compartir sus experiencias con problemas similares ya confrontados, pero siempre conservando una posición eminentemente técnica y auxiliar. La responsabilidad de la implementación descansa en manos de los grupos afectados.

—La intervención de un "tercer grupo" en cualquier situación experimental, tiene la función particular de estimular a los que están comprometidos en el proyecto a hacer explícitos sus objetivos, a documentar el curso seleccionado de los acontecimientos, a promover el intercambio de información y a dar su asistencia en el diseño de los aspectos técnicos implícitos en el esquema de evaluación seleccionado, pero sin obstaculizar la tarea principal de liberar las energías creativas de los involucrados.

Las "soluciones" impuestas inhiben la creatividad y sólo sirven para prolongar las dependencias tradicionales.

-Al examinar la variedad de trabajo experimental, tanto dentro de la red de la Fundación como fuera de ella, es interesante anotar que surge un patrón que indica que el entrenamiento específico del director de un proyecto afecta significativamente el carácter del enfoque seleccionado.

- La necesidad de una diversidad de opciones que respondan a requerimientos varios (por ejemplo de los niños o de los padres, o de ambos) se aprecia especialmente en soluciones de deprivación extrema o de cambio social rápido. Esto puede promover la creación de centros de cuidado diario, grupos pre-escolares con participación del hogar y los programas dirigidos a padres y madres con el propósito de ser mejores padres y madres.

- La importancia creciente de la colaboración de la mujer en la fuerza de trabajo, y la demanda, también creciente, de su participación en programas preescolares, genera situaciones de conflicto cuya solución parece ser, una vez más, alternativas amplias y diversificadas.

-Respecto al problema de lo que puede y debe ser transferido, un punto de vista es que los proyectos deben ser tan flexibles como sea posible y los modelos solo pueden ser creados después de un tiempo relativamente largo de funcionamiento. Una segunda opinión es que los problemas de la región requieren soluciones tan inmediatas que los proyectos deben desarrollarse rápidamente como respuesta a las necesidades de la comunidad. El punto de equilibrio puede encontrarse si las personas que ejecutan los proyectos tienen éxito en concretar sus metas, éxitos y errores, en forma tal, que otros pueden entenderlas y beneficiarse con su experiencia.

-El desarrollo de modelos viables, se hará en tres etapas: Una investigación inicial que estudiará la factibilidad de los objetivos del programa con base en un diseño experimental. Continuación de la investigación y desarrollo de materiales de entrenamiento y procedimiento para quienes implementen el modelo en contextos reales. Implementación del modelo en un contexto representativo. Esto último plantea un problema ético: Se debe utilizar un modelo en una comunidad diferente, cuando no se puede estar seguro de sus efectos? 
-El aspecto de técnicas y principios de evaluación es muy controvertido. La importancia de la evaluación es indiscutible, particularmente cuando se desea que el impacto de las innovaciones pueda ser entendido; sin embargo existe una gran limitación: la adecuación de las técnicas, considerando los antecedentes culturales de aquellos que son evaluados.

- Los criterios de un buen sistema de evaluación puede lograrse con la metodología de participación utilizada para la formulación de objetivos y metas. Un proyecto debe ser evaluado primero en términos de sus propias intenciones. Es necesario revisar la tendencia humana a ver sólo aquello que deseamos ver o lo que tememos, y planear un intento objetivo que demuestre que podemos estar equivocados. Un proyecto puede, o no, lograr sus objetivos explícitos pero casi siempre producirá logros inesperados y consecuencias no buscadas.

- Se espera que el contenido de programas educativos innovadores cambie los modelos existentes. Cada día es más evidente que el éxito de la interacción y el cambio social no depende de los aspectos cognoscitivos sino de la ecología total de las relaciones entre los individuos y la sociedad.

Rebeca Bernal Zapata 